\title{
纳米 $\gamma-\mathrm{Fe}_{2} \mathrm{O}_{3}$ 的结构相变 ${ }^{*}$
}

\author{
叶锡生 沙 健 方挥陈 斌 焦正宽 \\ (浙江大学物理系, 杭州 310027) \\ 吕光烈
}

(杭州大学中心实验室, 杭州 310028)

彭子飞 张立德

(中国科学院固体物理研究所,合肥 230031)

\section{关键词 纳米 $\gamma-\mathrm{Fe}_{2} \mathrm{O}_{3}$ 差热分析 (DTA) XRD 微结构 结构相变}

近十几年中发展起来的纳米固体材料因其独特的物理、化学、力学性质和在众多领域的应 用可能性而受到日益广泛的重视. 热稳定性是材料的一项重要性质. 在针对纳米材料热稳定 性所进行的研究中, 已经取得一些很有意义的结果 ${ }^{[1-4]}$, 例如, 发现不少纳米材料的热分析特 性曲线上存在一个放热峰. 但是, 对于此放热峰的起因尚存有异议, 有的认为是因晶粒长大而 引起, 而有的则归结于应力释放和界面原子的弛豫 ${ }^{[2]}$, 他们在所研究的样品中都没有发现此 峰与相变有关联.

我们在对所制备的纳米 $\gamma-\mathrm{Fe}_{2} \mathrm{O}_{3}$ 样品进行差热分析(DTA) 时发现, 其 DTA 曲线上也出现 了一不可重现的放热峰. 利用 $\mathrm{X}$ 射线衍射 (XRD) 对原始样品和一系列热处理样品的物相和 晶粒度进行了细致分析, 发现了此放热峰完全对应于从 $\gamma-\mathrm{Fe}_{2} \mathrm{O}_{3}$ 向着 $\alpha-\mathrm{Fe}_{2} \mathrm{O}_{3}$ 的结构相变; 这 一相变促进了晶粒生长过程, 但晶粒生长发生在比放热峰更宽的温区内, 我们认为放热峰主要 是由结构相变引起的, 在高于放热峰的温区内存在着 $\alpha-\mathrm{Fe}_{2} \mathrm{O}_{3}$ 晶粒的正常生长过程. 样品中 即使存在应力释放和界面原子弛豫所产生的放热过程, 它也是处于放热峰温区之内、与相变同 时发生的.

\section{1 实验方法}

采用液相法 ${ }^{[5]}$ 制备得到纳米 $\gamma-\mathrm{Fe}_{2} \mathrm{O}_{3}$ 样品 (中国科学院固体物理研究所). 差热分析工作 是在北京光学仪器厂生产的 WCT-2 型微机差热天平上进行的, 其温度扫描速度范围为 0 $99.9^{\circ} \mathrm{C} / \mathrm{min}$, 每次进行热分析测量时所使用的样品重量为 $15 \mathrm{mg}$ 左右. 对样品的 $\mathrm{X}$ 射线衍射 (XRD)物相分析和晶粒度分析采用日本产 Rigaku D/max-III B 型全自动衍射仪进行, 选用 $\mathrm{CuK} \alpha$ 辐射, 仪器功率为 $35 \times 25 \mathrm{~mA}$. 依据获得的 $\mathrm{XRD}$ 数据, 查对标准图谱可以确定样品的物 相, 由 $\mathrm{X}$ 射线线宽法 ${ }^{[5]}$ 亦即 Scherrer 公式可计算出样品的平均晶粒度.

199-01-03 收稿, 1996-04-16 收修改稿

* 国家自然科学基金资助项目 


\section{2 结果与讨论}

纳米 $\gamma-\mathrm{Fe}_{2} \mathrm{O}_{3}$ 差热分析结果如图 1 所示. 由图 1(a) 可见, 在以 $10^{\circ} \mathrm{C} / \mathrm{min}$ 的速度对纳米 $\gamma$ $\mathrm{Fe}_{2} \mathrm{O}_{3}$ 样品进行第 1 次温度扫描所得 DTA 曲线上, $440 \sim 550^{\circ} \mathrm{C}$ 的温度范围内出现了一显著的 放热峰(峰位在 $500^{\circ} \mathrm{C}$ 左右), 但相对应的热重 TG 曲线和 DTG 曲线上均无任何变化. 改变温 度扫描速度,此放热峰仍维持其形状. 对同一样品, 在同样测试条件下进行第 2 次升温扫描时 此放热峰不再出现, 如图 1(b)中所示. 这说明, 此放热峰是不可重现的.
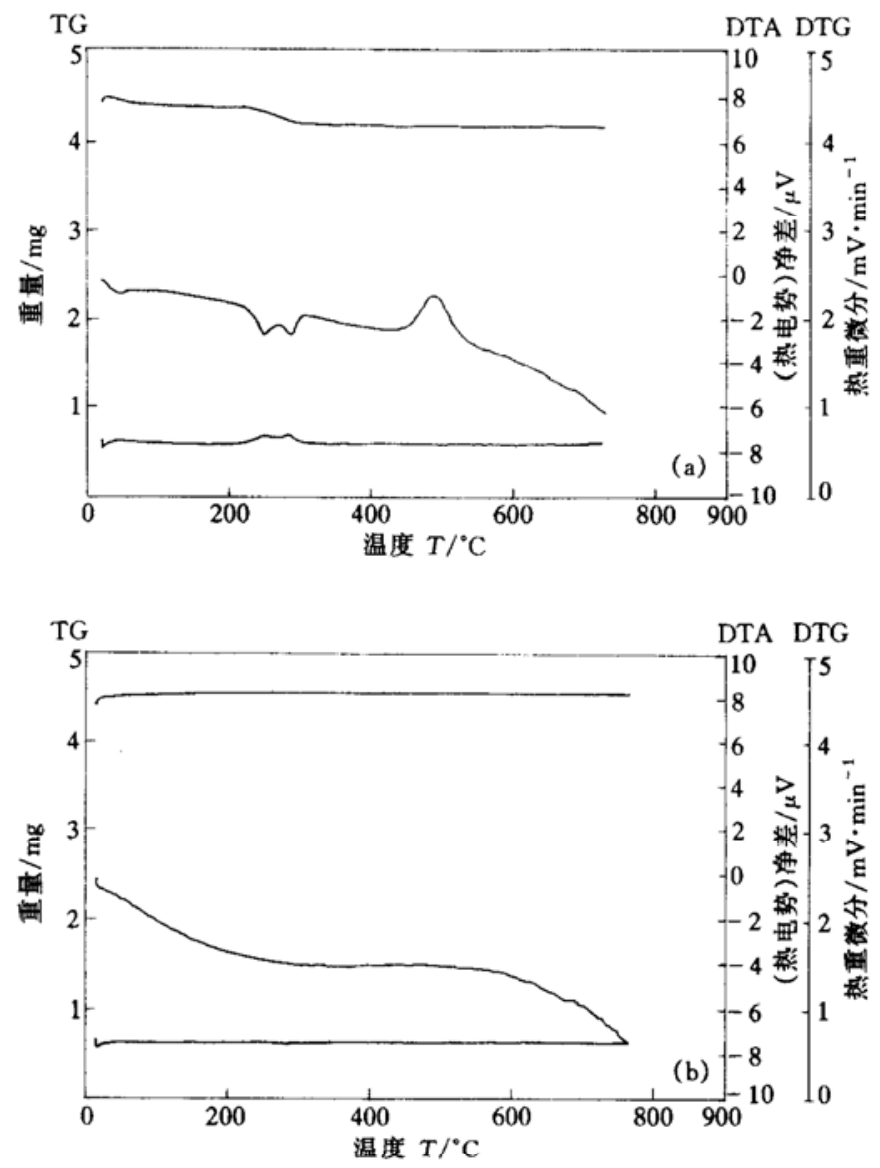

图 1 纳米 $\gamma-\mathrm{Fe}_{2} \mathrm{O}_{3}$ 的 TG-DTA 测量结果

扫描速度均为 $10 \mathrm{C} / \mathrm{min}$. (a) 纳米 $\gamma-\mathrm{Fe}_{2} \mathrm{O}_{3}$ 样品第 1 次升温测量的结果 (出现放热峰), (b) 对 (a)中同一样品进 行第 2 次差热测量(放热峰不再出现)

另外, 纳米 $\gamma-\mathrm{Fe}_{2} \mathrm{O}_{3}$ 的 DTA 曲线在 $200^{\circ} \mathrm{C}$ 以下的温区内出现了两个吸热峰, 与其相对应, 热重 TG 线上出现了两次失重. 我们认为这大多是由杂质(如水分子等)物理或化学吸附的脱 除过程所引起, 当然也不排除其他机制存在的可能性. 对这种吸热峰的形成原因, 我们将另作 进一步研究.

我们分别对纳米 $\gamma-\mathrm{Fe}_{2} \mathrm{O}_{3}$ 的原始样品 ( $1^{\#}$ )、升温至 $4200^{\circ} \mathrm{C}$ (放热峰之前) 然后淬火到室温 的样品 $\left(2^{\#}\right.$ )、升温至 $466^{\circ} \mathrm{C}$ (放热峰峰温处), 然后淬火到室温的样品 $\left(3^{\#}\right.$ )、升温至 $578^{\circ} \mathrm{C}$ (放热 峰消失), 然后淬火到室温的样品 $\left(4^{\#}\right)$ 和升温至 $728^{\circ} \mathrm{C}$ (远离放热峰), 然后淬火到室温的样品 
$\left(5^{\#}\right)$ 做了 XRD 实验, 并对 $1^{\#}, 2^{\#}, 4^{\#}$ 和 $5^{\#}$ 样品 进行了晶粒度分析. 其中, XRD 结果如图 2 所

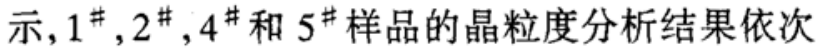
是 $12,12,35$ 和 $53 \mathrm{~nm}$.

由图 2 中 (a) 和 (b) 可知, 纳米 $\gamma-\mathrm{Fe}_{2} \mathrm{O}_{3}$ 样品 的结构在放热峰前保持为 $\gamma$ 相. 而图 2(c) 则表 明, 在放热峰出现的温区内, 样品处于 $\gamma-\mathrm{Fe}_{2} \mathrm{O}_{3}$ 与 $\alpha-\mathrm{Fe}_{2} \mathrm{O}_{3}$ 共存的混合相. 从图 2(d) 和 (e) 可以 看出, 样品在放热峰之后已全部转变为 $\alpha$ 相. 显 然, DTA 曲线上的放热峰完全对应着纳米 $\mathrm{Fe}_{2} \mathrm{O}_{3}$ 从 $\gamma$ 相到 $\alpha$ 相的转变.

结合以上差热分析、XRD 物相分析和晶粒 度分析的结果, 得到: 在热处理过程中, 放热峰出 现之前纳米 $\gamma-\mathrm{Fe}_{2} \mathrm{O}_{3}$ 样品维持原相且晶粒度几 乎不发生什么变化, 而在相变期间 (与放热峰相 对应)和相变之后晶粒度则发生了很大的变化 晶粒迅速长大．在高于放热峰的很宽温区 内, 晶粒仍在继续长大 (但相对生长速度有所下 降). 我们认为这是比 $\gamma-\mathrm{Fe}_{2} \mathrm{O}_{3}$ 晶粒更稳定的 $\alpha$ $\mathrm{Fe}_{2} \mathrm{O}_{3}$ 的正常长大过程, 但这一过程始自相变的 发生. 所以, 放热峰主要由结构相变引起, 而且 这一相变同时也促进了晶粒的生长过程.

纳米材料是具有亚稳态结构的材料, 在足够 高的温度下其颗粒将形成相对稳定的相并放出 热量. 在纳米 $\gamma-\mathrm{Fe}_{2} \mathrm{O}_{3}$ 中氧呈 fcc 密堆积, 为尖 晶石型结构. 在温度升到一定程度时, 纳米 $\gamma$ $\mathrm{Fe}_{2} \mathrm{O}_{3}$ 原有的亚稳结构状态被破坏, 畸变减小, 放出热量; 在放热峰出现之后, 样品已完全变为 结构状态相对稳定、畸变较小的 $\alpha-\mathrm{Fe}_{2} \mathrm{O}_{3}$, 其结构 中氧呈 hcp 密堆积. 在此结构相变过程中晶粒 也快速长大.

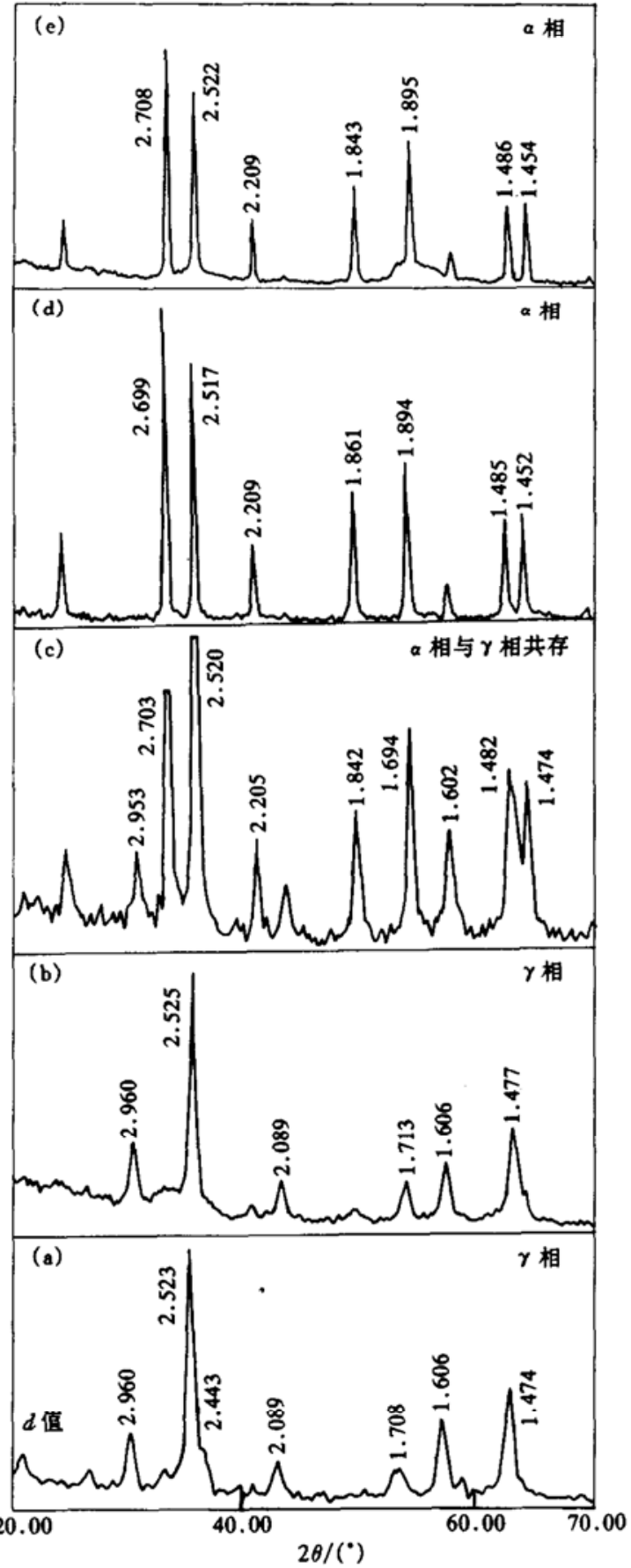

图 $2 \mathrm{X}$ 射线衍射(XRD)图谱

相变有助于晶粒长大这一点在 Hague 和 (a)纳米 $\gamma-\mathrm{Fe}_{2} \mathrm{O}_{3}$ 原始样品 ( $\left.1^{\#}\right),($ b $) 420 \mathrm{C}$ 淬火样品 ( $2^{\#}$ ), Mayo 对纳米 $\mathrm{TiO}_{2}$ 进行的研究结果 ${ }^{[6]}$ 中也同样 得到了证实.

(c) $466^{\circ}$ C 淬火样品 $\left(3^{\#}\right),(d) 578^{\circ} \mathrm{C}$ 淬火样品 $\left(4^{\#}\right)$, (e) $728^{\circ} \mathrm{C}$ 淬火样品 $\left(5^{\#}\right)$

\section{3 结论}

我们发现, 纳米 $\gamma-\mathrm{Fe}_{2} \mathrm{O}_{3}$ 样品的 DTA 曲线上存在一不可复现的放热峰. 通过 XRD 微结 
构分析,证实: 此放热峰完全对应于从 $\gamma-\mathrm{Fe}_{2} \mathrm{O}_{3}$ 向 $\alpha-\mathrm{Fe}_{2} \mathrm{O}_{3}$ 的结构相变;伴随着这一结构相变的 发生, 纳米 $\mathrm{Fe}_{2} \mathrm{O}_{3}$ 晶粒随温度上升而逐渐长大; 在高于放热峰的温区, 存在着 $\alpha-\mathrm{Fe}_{2} \mathrm{O}_{3}$ 晶粒的 正常长大过程. 在此结构相变过程中, 处于亚稳态的纳米 $\gamma-\mathrm{Fe}_{2} \mathrm{O}_{3}$ 转变为结构较稳定、畸变较 小的 $\alpha-\mathrm{Fe}_{2} \mathrm{O}_{3}$. 我们认为, 此放热峰主要是由结构相变而不是由应力释放或界面原子弛豫引起 的.

\section{参考文献}

1 Qin X Y, Wu X J, Zhang L D. The microhardness of nanocrystalline silver. Nanostructured Materials, 1995, 5(1):101 110

2 朱 勇. 纳米固体钼、氮化二钿、钝、氮化钛的 DSC 分析. 科学通报, 1994,39(5):469

3 孟进芳, 邹广田, 崔启良. 纳米晶钝酸铅体系的软模及相变研究. 中国科学, A 辑, 1995, 25(3):288

4 王锦辉, 陆怀先, 陈允鸿等. $88 \mathrm{Ni}-\mathrm{Fe}$ 合金超细微粒的制备、磁性以及热分析. 物理学报, 1995,44(8):1292

5 张立德, 牟季美. 纳米材料科学. 沈阳: 辽宁科学技术出版社, 1994. 93 102

6 Hague D C, Mayo M J. Effect of crystallization and phase transformation in nanocrystalline $\mathrm{TiO}_{2}$. Nanostructured Materials, $1993,3(3): 61 \sim 67$ 